\title{
Management of Lumbo-Peritoneal Shunt Complications in Patients with Idiopathic Intracranial Hypertension
}

\author{
Ibrahim Ewaiss ${ }^{1}$, Mohamed Rashid ${ }^{2}$, Al-Sayed Roshdy ${ }^{1}$, Samer Magdy $^{1 *}$ \\ Departments of ${ }^{1}$ Neurosurgery and ${ }^{2}$ Clinical Pathology, Faculty of Medicine, Al-Azhar University \\ * Corresponding Author: Samer Magdy Sharaf El-Din, E-mail: smmms2008@gmail.com
}

\begin{abstract}
Background: lumboperitoneal (LP) shunt is one of the best surgical management in treatment of Idiopathic intracranial hypertension (IIH).
\end{abstract}

Objective: to review the LP shunt complications and their management in IIH.

Patients and Methods: a total of 20 patients who got complications after LP shunt and needed shunt revision were included. There were 18 females and 2 males treated at Al-Hussein university hospital and Al-Mataria teaching hospital during the period from December 2015 and June 2018.

Results: there were $18(90 \%)$ females and $2(10 \%)$ males with age ranged from 18 to 48 years. Obstruction was the most common complication happened in $8(40 \%)$ patients. Migration was the second common complication happened in $6(30 \%)$ patients. Shunt infection is the one of the most dangerous complication and needs early intervention. There was one $(5 \%)$ case of infection which required removal of the LP shunt. Tow (10\%) patients had CSF leakage. One (5\%) patient had symptoms of over drainage. One (5\%) patient had radiculopathy. One $(5 \%)$ patient had intestinal perforation.

Conclusion: LP shunts for the treatment of IIH seems to be a safe procedure. Serious complications were rare. Only one case was deteriorated. Some patients need more than one shunt revision.

Keywords: Lunboperitoneal shunt, Idiopathic intracranial hypertension, complications.

\section{INTRODUCTION}

Idiopathic intracranial hypertension (IIH) is a disorder characterized by increased intracranial pressure with normal cerebrospinal fluid composition, and no other cause of intracranial hypertension evident on neuroimaging. Main symptoms are headache, vomiting, diplopia and visual disturbances ${ }^{(\mathbf{1})}$. Main signs are papilledema, visual field loss and sixth nerve palsy ${ }^{(2)}$.

The incidence of IIH is $0.9 / 100,000$ individuals. It increases to $3.5 / 100,000$ in women ages $15-44$, and to $19.3 / 100,000$ in women aged $20-44$ years who are $20 \%$ or more above their ideal weight ${ }^{(3)}$.

The treatment of IIH has two major goals: the alleviation of symptoms (usually headache) and the preservation of vision. Medical therapy and weight reduction are successful treatments in most patients. Visual fields should be monitored. In rare cases, visual defects or acuity may worsen despite medical treatment, and surgical treatment may be necessary. Surgical management ranges from repeated lumbar punctures to shunt operation and optic nerve sheath decompression ${ }^{(4)}$.

Lumboperitoneal (LP) shunt has many advantages over ventriculoperitoneal (VP) shunt includes: lack of cerebral cannulation, the possibility of using regional anesthesia with percutaneous insertion in adults, freedom from proximal obstruction by choroid plexus and brain parenchyma, a low rate of infection and the ability of insertion in the presence of small/slit ventricles ${ }^{(5)}$.

\section{AIM OF THE STUDY}

It is to evaluate the LP shunt complications and their management in $\mathrm{IIH}$.

\section{PATIENTS AND METHODS}

In this retrospective and prospective study, 20 patients with IIH got complications after LP shunt insertion (age ranged from 18 48 years with average of 31 years). There were 18 females and 2 males treated at Al-Hussein university hospital and Al-Mataria teaching hospital during the period from December 2015 and June 2018. Written consent was obtained 
from all patients or their legal representative. A detailed history was taken and thorough physical examination was performed in all cases. Pre-operative investigation was done according to the complication. Post-operative follow ups at 1,3,6 months by clinical data, visual examinations and neuroimaging.

\section{- Inclusion criteria:}

- Patients that received primary placement of LP shunt as treatment of IIH.

- $\quad$ Adult Patients (age >18).

- Needs surgical management.

\section{- Exclusion criteria:}

- Conditions other than IIH treated with LP shunts.

- Non-primary placed LP shunts.

- Pediatric patients.

- Pregnancy.

- Ethical approval:

The study was approved by the Ethics Board of Al-Azhar University.

\section{- Confidentiality:}

The data of the participant were recorded and analyzed without any personal identifiers, by using coded information. The source documents and identification lists were archived in a secured facility per center. Permission for accessing data will be documented per investigator.

\section{- Preoperative protocol includes:}

- Complete general and neurological examination.

- Routine preoperative investigations.

- Plain radiographs of the Lumbar region antero-posterior and lateral views.

- Magnetic resonance imaging (MRI).

- Fundus examination.

- Visual field test.

- Post-operative protocol includes:

- Regular follow ups at 1,3,6 months.

- Clinical data.

- Fundus examination and visual field.

- Radiological imaging:

- CT or MRI brain.

- X-Ray lumbar region.

\section{- Statistical analysis:}

Recorded data were analyzed using the statistical package for social sciences, version 20.0 (SPSS Inc., Chicago, Illinois, USA). Quantitative data were expressed as mean \pm standard deviation (SD). Qualitative data were expressed as frequency and percentage.

\section{RESULTS}

Twenty patients were identified (18 women and 2 men). The average age of these patients was 31 years. Most patients were obese. Headache was the most common presenting symptom. Papilledema is the most important physical finding in patients with IIH (Table 1).

Table (1): Patient characteristics:

\begin{tabular}{|l|l|}
\hline Variables & No. $(\%)$ \\
\hline Age range (years) & $18-48$ \\
\hline Male: Female & $1: 9$ \\
\hline Obese & $17(85 \%)$ \\
\hline Anemia & $7(35 \%)$ \\
\hline Diabetes mellitus & $4(20 \%)$ \\
\hline Contraceptive pills & $4(20 \%)$ \\
\hline Headache & $18(90 \%)$ \\
\hline Blurring of vision & $16(80 \%)$ \\
\hline Papilledema & $17(85 \%)$ \\
\hline Visual field affection & $16(80 \%)$ \\
\hline
\end{tabular}

Regarding complications (Table 3):

In our series shunt obstruction was the most common complication of LP shunt as it presents in 8 patients (40\%). Diagnosis of obstruction was done by recurrence of the symptoms and signs and normal imaging. Unfortunately, the diagnosis confirmed only intra-operative. The most common site of complication was the peritoneal catheter.

Shunt migration were found in 6 patients (30\%). Migration of the peritoneal catheter may lead to pseudocyst formation. Shunt migration can easily diagnose by radiological imaging.

Shunt infection is one of the most dangerous complications and meningitis is the one potentially life-threatening complication of the LP shunt. In our series, one patient had wound infection $(5 \%)$ and treated by removal of shunt and intra-venous antibiotics.

Intestinal perforation, a very rare complication, happened in one patient with the use of percutaneous technique.

Table (2): LP shunt Complications: 


\begin{tabular}{|l|l|l|}
\hline Complication & Frequency & Percent \\
\hline Shunt obstruction & 8 & $40 \%$ \\
\hline Shunt migration & 6 & $30 \%$ \\
\hline Over drainage & 1 & $5 \%$ \\
\hline CSF Leakage & 2 & $10 \%$ \\
\hline Infection & 1 & $5 \%$ \\
\hline Radiculopathy & 1 & $5 \%$ \\
\hline $\begin{array}{l}\text { Intestinal } \\
\text { Perforation }\end{array}$ & 1 & $5 \%$ \\
\hline
\end{tabular}

Regarding outcome (Table 3):

$80 \%$ of patients achieved good symptoms improvement. Only one patient deteriorated due to infection.

Table (3): Outcome of shunt revision:

\begin{tabular}{|l|l|l|l|}
\hline Variables & Improve & Same & $\begin{array}{l}\text { Deteriorate } \\
\text { d }\end{array}$ \\
\hline Obstruction & 6 & 2 & 0 \\
\hline Migration & 6 & 0 & 0 \\
\hline CSF Leakage & 1 & 1 & 0 \\
\hline Over drainage & 1 & 0 & 0 \\
\hline Infection & 0 & 0 & 1 \\
\hline Radiculopathy & 1 & 0 & 0 \\
\hline $\begin{array}{l}\text { Intestinal } \\
\text { Perforation }\end{array}$ & 1 & 0 & 0 \\
\hline $\begin{array}{l}\text { Total } \\
\text { (percent) }\end{array}$ & $\mathbf{1 6}(\mathbf{8 0 \% )}$ & $\begin{array}{l}\mathbf{3} \\
(\mathbf{1 5 \% )}\end{array}$ & $\mathbf{1 ( 5 \% )}$ \\
\hline
\end{tabular}

\section{DISCUSSION}

LP shunts have been demonstrated to be effective in the treatment of IIH. Although LP shunt has many advantages over VP shunt, LP shunt associated with number of complications $^{(2)}$. However, serious complications are rare. This study included 20 patients with IIH and got complications after placement of LP shunt. There were 18 females (90\%), 2 males with a ratio of 9:1. The female predominance was found in the series of Kalyvas et al. ${ }^{(6)}$ who reported that females representing $90.1 \%$ of the patients in their study. Also, Yadav et al. ${ }^{(7)}$ reported $91.6 \%$ were females and Eggenberger et al. (8) reported $88 \%$ were females.

In our study, $17(85 \%)$ patients were obese. The strong association of IIH with obesity has been reported in many previous studies, but the pathophysiologic mechanism of obesity and increased intracranial pressure is undetermined. Kalyvas et $\boldsymbol{a l} .{ }^{(6)}$ reported that $81.7 \%$ were obese patients. Also, Jones et al. ${ }^{(9)}$ reported that $67 \%$ were obese and Eggenberger $\boldsymbol{e t}$ al. ${ }^{(8)}$ reported that $88.8 \%$ were obese.
In our series, shunt obstruction was the most common complication of LP shunt as it presents in 8 patients (40\%). Diagnosis of obstruction was done by recurrence of the symptoms and signs and normal imaging. Unfortunately, the diagnosis confirmed only intra-operative. Kalyvas et al. ${ }^{(6)}$ reported shunt obstruction in $51.6 \%$ of operated patients.

Shunt infection is one of the most dangerous complications and meningitis is the one potentially life-threatening complication of the LP shunt. In our series, one patient had wound infection (5\%) and treated by removal of shunt and intra-venous antibiotics. Wang et al. (10) reported shunt infection in $1.8 \%$ of operated patients.

In fact, over drainage is a common complication of LP shunt but most of symptoms are mild and no surgical management is needed. So, only one (5\%) in our serious developed over drainage and treated by higher pressure shunting. Significant over drainage may lead to serious complication as subdural collection and acquired Chiari malformation. Jusué-torres et al. (12) reported over drainage in $20 \%$ of patients. Barash et al. ${ }^{(11)}$ reported a rare case of bilateral subdural hematoma.

Radiculopathy and myelopathy were reported as a complication for LP shunt and they usually occur in adult patient. Radicular pain maybe resolved spontaneously within 3 weeks but if persist revision is necessary but myelopathy occurs in the form of paraparesis which need urgent revision. In our series, one patient $(5 \%)$ had radicular pain and treated by reposition of the shunt. Jusué-torres et al. ${ }^{(12)}$ reported radiculopathy in $8 \%$ of patients.

\section{CONCLUSION}

Shunt obstruction was the most common complication. Unfortunately, diagnosis confirmed only intra-operative. Shunt migration was the second most common complication and the investigation of choice was C-T. The most dangerous complication was infection and needs urgent management. In cases with failed LP shunt, CSF diversion could be preceded by VP shunt or optic nerve sheath fenestration. Intestinal perforation was happened with the use of percutaneous technique. So, we recommend open surgery and laparoscopic technique. 
Overall, LP shunts for the treatment of IIH seems to be a safe procedure. Serious complications were rare. Only one case was deteriorated. Some patients need more than one shunt revision.

\section{REFERENCES}

1. Victorio M C, Rothner A D, (2013): Diagnosis and treatment of idiopathic intracranial hypertension (IIH) in children and adolescents. Curr Neurol Neurosci Rep., 13: 336-342.

2. Yadav Y R, Pande S, Raina V K, Singh M (2004): Lumboperitoneal shunts: review of 409 cases. Neurol India, 52: 188-190.

3. Hannerz J, Ericson $K$ (2009): The relationship between idiopathic intracranial hypertension and obesity. Headache, 49: 178-184.

4. Julayanont $P$, Karukote A, Ruthirago D, Panikkath D, Panikkath R (2016): Idiopathic intracranial hypertension: Ongoing clinical challenges and future prospects. Journal of Pain Research, 9: 87-99.

5. Wall M (2010): Idiopathic intracranial hypertension. Neurol Clin., 28: 593617.
6. Kalyvas A, Liouta E (2016): Idiopathic Intracranial Hypertension: Epidemiology, pathophysiology, clinical features and contemporary management. Chronicles, 11: 77-84.

7. Yadav Y R, Parihar V, Agarwal M, Bhatele P R, Saxena N (2012): Lumbar peritoneal shunt in idiopathic intracranial hypertension. Turk. Neurosurg, 22: 21-26.

8. Eggenberger $\mathbf{E}$ R, Miller $\mathbf{N} \mathbf{R}$, Vitale $S$ (1996): Lumboperitoneal shunt for the treatment of pseudotumor cerebri. Neurology, 46: 1524-1530.

9. Jones J S, Nevai J, Freeman M P, McNinch D E (1999): Emergency department presentation of idiopathic intracranial hypertension. Am J Emerg Med., 17: 517-521.

10. Wang V Y (2007): Complications of lumboperitoneal shunts. Neurosurgery, 60: 1045-1048.

11. Barash I A, Medak A J (2013): Bilateral subdural hematomas after lumboperitoneal shunt placement. J Emerg Med., 45: 178-181.

12. Jusué-torres I, Hoffberger $\mathbf{J} \quad \mathrm{B}$, Rigamonti D (2015): Complications Specific to Lumboperitoneal Shunt. Springer Cham., 2015: 203-211. 